\title{
Identifying and utilizing the ischemic penumbra
}

Marc Fisher, MD

Birgul Bastan, MD

Correspondence \& reprint requests to Dr. Fisher: fisherm@ummhc.org

\section{ABSTRACT}

The penumbral concept is defined as different areas within the ischemic region evolve into irreversible brain injury over time and that this evolution is most critically linked to the severity of the decline in cerebral blood flow (CBF). The ischemic penumbra was initially defined as a region of reduced CBF with absent spontaneous or induced electrical potentials that still maintained ionic homeostasis and transmembrane electrical potentials. The reduction of CBF levels to between 10 and $15 \mathrm{~mL} / 100 \mathrm{~g} / \mathrm{min}$ and approximately $25 \mathrm{~mL} / 100 \mathrm{~g} / \mathrm{min}$ are likely to identify penumbral tissue, and the ischemic core of irreversible ischemic tissue has a CBF value below the lower threshold. The role of identifying this critically deprived brain tissue from CBF in triaging patients for endovascular ischemic therapy is evolving. In this review we focus on the basic science of the penumbral concept and identification using various imaging modalities (PET, MRI, and CT) in animal models and human studies. Another article in this supplement addresses the clinical implication and the current understanding and application of this concept into clinical practice of endovascular ischemic stroke therapy. Neurology ${ }^{\circledR} 2012 ; 79$ (Suppl 1):S79-S85

\section{GLOSSARY}

$\mathbf{A D C}=$ apparent diffusion coefficient; $\mathbf{C B F}=$ cerebral blood flow; $\mathbf{C B V}=$ cerebral blood volume; $\mathbf{C M R O}_{2}=$ cerebral metabolic rate of oxygen; DEFUSE = Diffusion and Perfusion Imaging Evaluation For Understanding Stroke Evolution; DIAS II = Desmoteplase in Acute Ischemic Stroke Trial; DWI = diffusion-weighted MRI; EPITHET = Echoplanar Imaging Thrombolytic Evaluation Trial; $\mathbf{M T T}=$ mean transit time; $\mathbf{O E F}=$ rate of oxygen extraction; $\mathbf{P W I}=$ perfusion-weighted $\mathbf{M R I} ; \mathbf{T}_{\max }=$ time to maximum concentration; $\mathbf{t P A}$ = tissue plasminogen activator.

The concept of the ischemic penumbra was initially proposed by Astrup et al. ${ }^{1}$ and represented an important milestone for understanding the temporal and spatial evolution of focal ischemic brain injury. The penumbral concept suggests that different areas within the ischemic region evolve into irreversible brain injury over time and that this evolution is most critically linked to the severity of decline in cerebral blood flow (CBF). The ischemic penumbra was initially defined as a region of reduced CBF with absent spontaneous or induced electrical potentials that still maintained ionic homeostasis and transmembrane electrical potentials. This narrow definition has evolved, and 2 subsequent definitions should be focused upon. Hossmann ${ }^{2}$ characterized the ischemic penumbra as "a region of constrained blood supply in which energy metabolism is preserved." In an accompanying editorial, Ginsberg and Pulsinelli ${ }^{3}$ modified this definition, suggesting that energy metabolism might be intermittently compromised within the ischemic penumbra. If the ischemic penumbra is characterized by these approaches, then a reduction of CBF to levels between a lower threshold of $10-15 \mathrm{~mL} / 100 \mathrm{~g} / \mathrm{min}$ and an upper threshold of approximately $25 \mathrm{~mL} / 100 \mathrm{~g} / \mathrm{min}$ is likely to identify penumbral tissue. The ischemic core of infarcted tissue has a CBF value below the lower threshold. The upper threshold for $\mathrm{CBF}$ decline approximates that level associated with the development of neurologic deficits and is substantially less severe than the CBF decline associated with loss of electrical activity, as proposed by the original ischemic penumbra concept.

The pathophysiologic relationship of $\mathrm{CBF}$ decline and metabolic activity in ischemic tissue is important but not easy to relate to the neurologic status of stroke patients. A simple but meaningful definition of the ischemic penumbra for both clinicians and clinical trialists was originally proposed by Hakim ${ }^{4}$ and states that the ischemic penumbra is ischemic tissue that is "potentially reversible" with a timely intervention. This concept was expanded by relating the potential for reversibility to how such ischemic tissue might be charac-

From the Department of Neurology, University of Massachusetts Medical School, Worcester, MA.

Go to Neurology.org for full disclosures. Disclosures deemed relevant by the authors, if any, are provided at the end of this article. 
terized by imaging and then treated. ${ }^{5}$ If reducing infarct size to improve functional outcome is the goal of acute stroke therapy and the ischemic penumbra is that portion of the ischemic injury that can potentially be reversed, then identifying it and mapping its evolution is of obvious importance.

After the earlier characterizations of the penumbra, refined definitions were proposed that focused on the molecular consequences of the CBF reduction in this region. A large number of cellular effects occur in penumbral tissue, including a reduction of protein synthesis with preservation of adenosine triphosphate synthesis, the induction of acidosis, the release of excitotoxic neurotransmitters, and increases in the levels of nitric oxide, interleukin-1, transforming growth factor- $1 \beta$, tumor necrosis factor $-\alpha$, vascular endothelial growth factor, brain-derived neurotrophic factor, and hypoxia inducible factor. ${ }^{6}$ Other molecules such as matrix metalloproteinases and NMDA-signaling molecules may have a dual role, mediating ischemic injury shortly after it is initiated but enhancing recovery mechanisms at later time points. ${ }^{7}$ Recent proteomic data demonstrated upregulation of proteins involved with antiapoptotic, anti-inflammatory, and mitochondrial heat shock activity in cell cultures exposed to ischemic conditions. ${ }^{8}$ This enhanced understanding of the cellular and molecular consequences associated with the penumbra could provide targets for the development of novel therapies. In addition to these advances concerning the cellular and molecular consequences of penumbral levels of CBF decline, involvement of the entire neurovascular unit and not just neurons within the penumbral region has gained acceptance and should also be considered in future therapy development. ${ }^{9}$

Initial imaging studies of the ischemic penumbra in both animal stroke models and humans were performed with PET by a few investigators. PET allows researchers to evaluate in vivo the relationship between CBF and such metabolic parameters as the cerebral metabolic rate of oxygen $\left(\mathrm{CMRO}_{2}\right)$ and the rate of oxygen extraction (OEF) by brain tissue. ${ }^{10} \mathrm{In}$ normal brain, $\mathrm{CBF}$ and $\mathrm{CMRO}_{2}$ have a linearly proportional relationship, and OEF is similar all over the brain. Four PET patterns are observed with focal brain ischemia. ${ }^{11}$ The first pattern is an increase in cerebral blood volume (CBV) to maintain $\mathrm{CBF}$ in response to physiologic conditions or demands (autoregulation). The second pattern is an increase of $\mathrm{OEF}$ in response to a reduction of $\mathrm{CBF}$ so that $\mathrm{CMRO}_{2}$ is maintained, and this pattern defines oligemia. The third PET pattern is an increase of OEF in brain regions with reduced $\mathrm{CBF}$ and $\mathrm{CMRO}_{2}$ to try to maintain tissue metabolism as much as possi- ble, and this pattern defines ischemic tissue with penumbral characteristics. The fourth pattern is very low $\mathrm{CBF}$ and $\mathrm{CMRO}_{2}$ levels with poor OEF, and this pattern represents irreversible ischemic injury or the infarct core. In both cats and baboons subjected to experimental focal ischemia and serial MRI studies, evolution of the ischemic lesion from a penumbral pattern to an infarct pattern occurred. ${ }^{12,13}$ PET studies in stroke patients have yielded valuable information about the location and temporal evolution of the ischemic penumbra. ${ }^{14}$ These studies have identified penumbral ischemic tissue many hours after stroke onset and suggest that that the time window for potentially effective therapy may be quite long in some patients.

Another PET approach for the potential identification of the ischemic penumbra is to use ligands that preferentially bind to penumbral tissue and not to irreversibly injured tissue. Two such ligands are ${ }^{11} \mathrm{C}$ flumazenil and ${ }^{18} \mathrm{~F}$-fluoromisonidazole. Both ligands bind preferentially to hypoxic but potentially viable ischemic tissue, and preliminary studies suggest they can be used to distinguish the ischemic penumbra and core. ${ }^{15,16}$ The problem with both PET metabolic and ligand imaging is the limited availability of PET scanners for routine clinical use and the time-consuming nature of the studies to be performed. PET will remain a useful research tool but will not be useful for clinical trials and patient care.

Diffusion-weighted imaging (DWI) and perfusionweighted MRI (PWI) are widely available MRI modalities that provide valuable information about the tissue characteristics of focal brain ischemia and flow in the microcirculation. ${ }^{17}$ Abnormalities of the apparent diffusion coefficient (ADC) in ischemic brain regions on DWI and tissue perfusion on PWI are detectable within minutes after the onset of ischemia in animal stroke models and in most stroke patients when they are initially imaged. This information is of obvious benefit for stroke diagnosis and localization, but it may also provide insights into the existence and size of potentially reversible ischemic tissue. It was initially proposed that ischemic brain regions with reduced perfusion on PWI and hyperintensity on DWI represented regions of infarction and that regions with abnormal perfusion that were normal on DWI represented the penumbra. ${ }^{18}$ This so-called DWI-PWI mismatch is clinically useful but only approximates the distinction between infarct core and penumbra (figure 1). Part of the PWI abnormality represents oligemic tissue where the severity of $\mathrm{CBF}$ decline is relatively mild, and this tissue will not become irreversibly injured. ${ }^{19}$ Conversely, portions of the DWI abnormality with relatively modest ADC declines can be reversed with timely reperfusion, as has been demonstrated in both experimental stroke models 


\section{Figure 1 Diffusion/perfusion MRI}

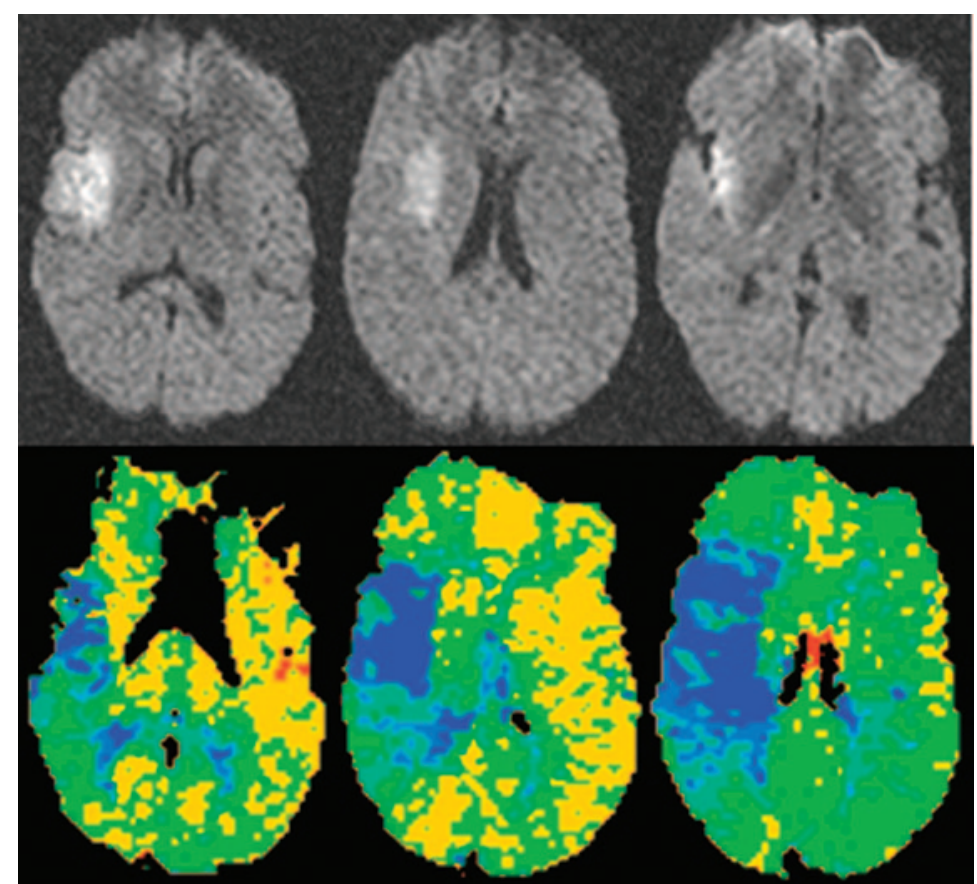

The top row of images shows a diffusion lesion in white on multiple levels; the bottom row shows the corresponding perfusion lesions at these levels.

and stroke patients undergoing both IV and intraarterial thrombolytic therapy..$^{20,21}$ An important variable in the identification of the DWI-PWI mismatch is how the 2 regions of abnormality are identified. DWI lesion identification is relatively straightforward as a region of hyperintensity corresponding to reduced ADC values. Characterizing the PWI region of abnormality is more problematic because it is dependent upon what threshold is used to define hypoperfused tissue. ${ }^{22}$ Currently, there is no well-validated, widely agreed upon threshold to define PWI abnormality, and proposals have been made to use maps of time to peak, mean transit time (MTT), and time to maximum concentration $\left(\mathrm{T}_{\max }\right)$ to define PWI hypoperfusion. Validation of the most appropriate and precise PWI identification of hypoperfused tissue remains to be established. Currently, the MTT and $\mathrm{T}_{\max }$ approaches are most widely used, and despite precise validation they allow clinicians to reasonably identify obvious mismatches between abnormal PWI regions and DWI hyperintense regions.

In experimental stroke studies the natural history and effects of treatment on the evolution of the DWI-PWI mismatch were evaluated. In experiments using validated thresholds to identify the diffusion and perfusion lesion volumes, the evolution of the mismatch in the rat suture occlusion and embolic stroke models demonstrated a larger and more persistent mismatch in the embolic model. ${ }^{23}$ This suggests that the embolic model is closer to the pathophysiology of human stroke than the suture model and has more potentially salvageable ischemic tissue that might be amenable to treatment for a longer time period. Treatment experiments demonstrated that granulocyte colony stimulating factor initiated 1 hour after stroke onset in the permanent occlusion suture model halted the enlargement of the DWI, maintaining the DWI-PWI mismatch for many hours, and reduced the extent of histologically confirmed infarction at 24 hours. ${ }^{24}$ In the embolic rat stroke model, high-flow normobaric hyperoxia also stopped the enlargement of the DWI lesion, and when combined with IV tissue plasminogen activator (tPA) at 3 hours after stroke onset, it allowed this therapy to significantly reduce infarct volume at 24 hours in comparison with a control group (room air and tPA at 3 hours). ${ }^{25}$ Characterizing penumbral tissue by using absolute ADC values and quantitative CBF measurements with arterial spin-labeling PWI appears to be more precise than the relatively simplistic approach with bolus contrast PWI. As this PWI technique becomes available for human stroke imaging, a similar approach to more precise penumbral identification will likely evolve.

Perfusion CT is the second imaging modality that is being used to identify an approximation of the penumbra in patients with acute ischemic stroke. Perfusion CT images are acquired on ultrafast CT scanners by rapid repetitive scanning after the injection of iodinated contrast material. With a signal washout curve generated by bolus-contrast perfusion CT scanning, semiquantitative CBV and CBV maps can be generated. ${ }^{26}$ As ischemia becomes more severe, CBV collapses, so with perfusion CT the ischemic core is identified as a region with a markedly reduced CBV. CBF or MTT maps demonstrate ischemic regions with values below a defined threshold as abnormally perfused brain regions. The ischemic penumbra is operationally defined as the region of CBF or MTT abnormality with CBV values above the threshold of collapse (figure 2). ${ }^{27,28}$ The use of perfusion CT is currently hampered by several factors, as compared with DWI-PWI. These factors are limited coverage of the ischemic brain (only 2-4 slices for most scanners, although whole brain coverage is now available on newer models), less reliably identified thresholds for identifying CBF/MTT and CBV abnormalities, and very little animal modeling work with this imaging technology. A major advantage of perfusion CT is the more widespread availability and access in comparison with MRI. A study with a modest sample size did demonstrate comparable identification of target patients for treatment decisions in the 3- to 9-hour time window with perfusion CT as compared with DWI-PWI, but this finding will need to be confirmed in many more pa- 


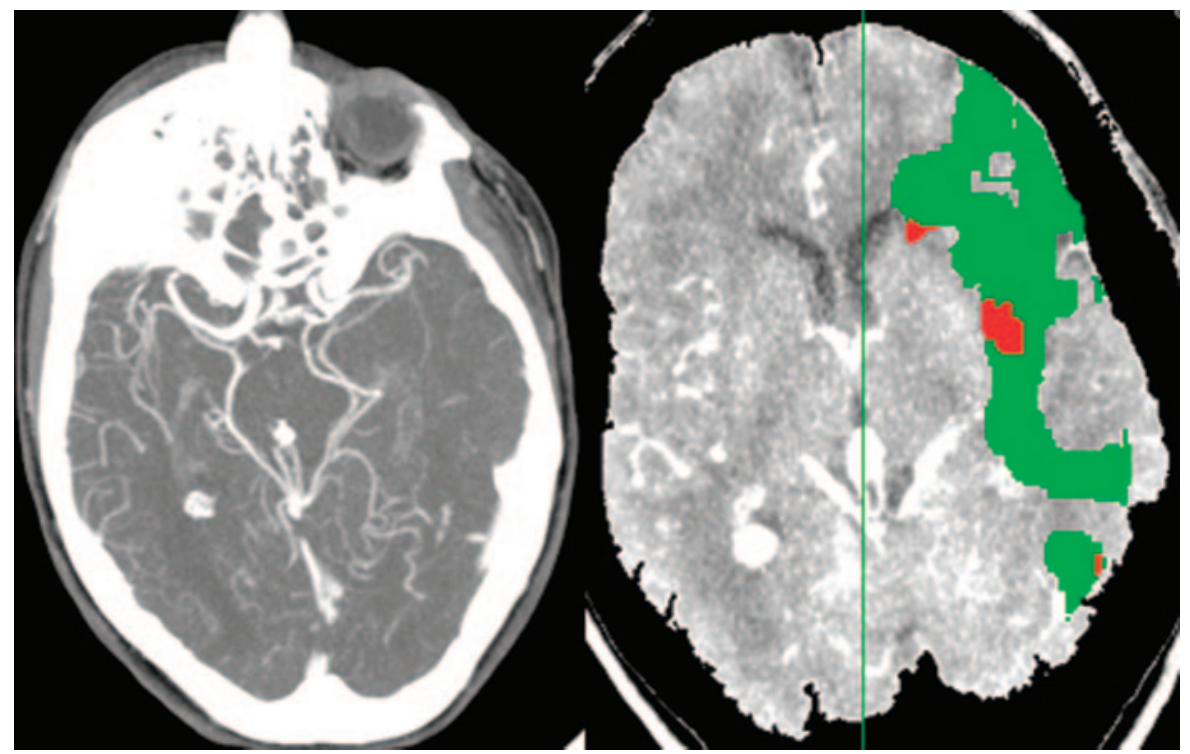

The CT angiogram shows occlusion of the middle cerebral artery and the perfusion CT scan shows a large mismatch between the mean transit time lesion (green) and the cerebral blood volume lesion (red).

tients at multiple sites. ${ }^{29}$ When some of the current problems with perfusion CT are solved and comparability to DWI-PWI for penumbral approximation is confirmed, practical considerations suggest that it will become the most commonly used imaging tool for penumbral approximation.

The potential utility of penumbral imaging for the selection of stroke patients to treat beyond the current 3-hour window of IV tPA is emerging from case series and early clinical trials. Two European case series of stroke patients with a DWI-PWI mismatch treated with IV between 3 and 6 hours after stroke onset demonstrated a favorable 90-day outcome rate comparable to that for patients treated in the 0 - to 3-hour time window who were screened only with a noncontrast CT scan. ${ }^{30,31}$ These case series did not have a control group of mismatch patients who did not undergo IV tPA treatment. In the Diffusion and Perfusion Imaging Evaluation For Understanding Stroke Evolution (DEFUSE) study, patients were treated with IV tPA in the 3- to 6-hour window, and all patients were studied with a comprehensive MRI battery that included DWI and PWI. ${ }^{32}$ A mismatch was not required for inclusion, but the primary hypothesis of this modestly sized exploratory study was that mismatch patients would respond better to therapy than non-mismatch patients. PWI abnormalities were identified by a $\mathrm{T}_{\max }$ delay of $>2$ seconds, and a greater than $20 \%$ mismatch of the PWI and DWI lesion volumes at baseline was observed in 37 (54\%) of 68 patients. Early reperfusion occurred in $49 \%$ of patients in whom it could be determined. This early reperfusion in mis- match patients was associated with a favorable outcome, defined as a modified Rankin Scale score of $0-2$ in $56 \%$ of patients, whereas only $19 \%$ of mismatch patients without reperfusion achieved this outcome. Some mismatch patients had a "malignant mismatch pattern," defined as a large $(>100 \mathrm{~mL})$ baseline DWI lesion or a PWI lesion $>100 \mathrm{~mL}$ with a very long, 8-second delay of $T_{\max }$. Such a malignant pattern was observed infrequently but was associated with a low rate of favorable outcome and a $50 \%$ risk of fatal intracerebral hemorrhage. In nonmismatch patients, absence of early reperfusion was associated with a higher rate of favorable outcome than with early reperfusion. Of those whose reperfusion was successful, non-mismatch patients had a less favorable outcome than mismatch patients. The number of each of these types of scenarios was quite small.

Another modestly sized study, the Echoplanar Imaging Thrombolytic Evaluation Trial (EPITHET), randomized patients to IV tPA or vehicle in the 3- to 6-hour time window and included baseline MRI assessment but not a requirement of mismatch for inclusion. ${ }^{33} \mathrm{~A} \mathrm{~T}_{\max }$ delay of $>2$ seconds was also used to define PWI abnormalities, as in DEFUSE, but in this study $86 \%$ of the 101 patients had a $20 \%$ or greater mismatch of baseline PWI and DWI volumes. The primary outcome measure of the study that was prespecified was infarct growth, assessed by the geometric mean relative growth; this was 1.24 in the TPA group and 1.78 in the placebo group $(p=$ $0.24)$. When ischemic lesion growth was dichotomized into growth or no growth between baseline and day $90,23 \%$ more of the tPA group had no 
growth, barely missing statistical significance. Reperfusion of more than $90 \%$ of the initial PWI lesion was observed in $56 \%$ of the tPA patients and $26 \%$ of the placebo patients $(p=0.01)$. Dichotomization of the modified Rankin Scale scores, at $0-1$ vs 2-6, yielded $15 \%$ more tPA patients who achieved a favorable outcome $(p=0.153)$, but if the dichotomization was $0-2$ vs 3-6, then the difference was only 5\%. A malignant mismatch pattern was not associated with an increased risk for bleeding. Recently, a pooled analysis of the DEFUSE and EPITHET datasets was performed, using the same automated imaging analysis program, RAPID, for both and the same threshold for defining the PWI lesion, a $\mathrm{T}_{\text {max }}$ delay of $>6$ seconds. In this combined analysis, target mismatch patients whose reperfusion was successful had an OR of 5.6 for a 90-day favorable outcome, as compared with such patients whose reperfusion was not successful. In non-mismatch patients, reperfusion was not observed to improve the chances for a favorable outcome. ${ }^{34}$

Desmoteplase, a thrombolytic agent derived from bat saliva, was also evaluated in 2 small trials that employed perfusion/diffusion MRI; a $>20 \%$ mismatch was required for inclusion. Desmoteplase doses of 90 and $125 \mu \mathrm{g} / \mathrm{kg}$ were shown to safely induce reperfusion, with a trend toward reducing infarct size and improving clinical outcome. ${ }^{35,36} \mathrm{~A}$ larger trial of desmoteplase, the Desmoteplase In Acute Ischemic Stroke Trial (DIAS II), studied these 2 doses vs placebo and demonstrated no clinical benefit with either desmoteplase dose and an increase in late mortality with the higher dose. ${ }^{37}$ In this study, approximately two-thirds of patients were included with a PWI-DWI mismatch $>20 \%$, and the other one-third were included on the basis of a CBF-CBV mismatch on perfusion CT. ${ }^{26}$ The trial did not evaluate reperfusion and had a clinical outcome at day 90 as the primary outcome assessment. Baseline stroke severity was modest in both active treatment groups and the placebo group; median NIH Stroke Score scale was 9 in all groups, and no difference in clinical outcome was seen at day 90. The higher-dose desmoteplase group had a tendency toward a worse outcome because of late mortality that was unlikely secondary to treatment. An analysis combining only the MRI-screened patients from DIAS II with the patients from the 2 smaller, earlier trials demonstrated that the percentage of patients achieving a modified Rankin Scale score of 0-2 was 13\% higher (almost statistically significant) with desmoteplase. ${ }^{38}$

The data from the case series of patients selected by MRI 3-6 hours after stroke onset, the 3- to 6-hour IV tPA alone DEFUSE study, the 3- to 6-hour comparison of IV tPA vs placebo in the
EPITHET study, and the 3 desmoteplase trials provide useful information for the design of future beyond-3-hour reperfusion trials. It does appear that selection of putative penumbra, as identified by MRI, as a substantial DWI-PWI mismatch in patients with acute ischemic stroke can identify those who are more likely to respond to reperfusion therapy over an extended time window. The sample size likely to be needed in phase II trials and in phase III trials to determine a beneficial clinical outcome will be modest: likely, several hundred patients per dose group, and certainly much less than would be needed for a trial without penumbral imaging. ${ }^{39}$

The precise definition of the PWI abnormality will require further validation, but a $T_{\text {max }}$ delay of more than the currently used 2 seconds will likely be necessary for more precise characterization of hypoperfused tissue destined for infarction. A mismatch percentage of greater than $20 \%$ will apparently be needed to identify a study population most likely to respond to treatment. The imaging and clinical inclusion/exclusion criteria will need to be harmonized to insure that the baseline stroke severity of patients randomized is not too mild, precluding a high rate of good outcomes in the placebo group. In addition to using a DWI-PWI mismatch approach in reperfusion-based clinical trials, such methodology could be used in future neuroprotection trials to enhance recruitment of patients more likely to respond to treatment, especially at delayed time points. ${ }^{40}$ Currently, perfusion CT is less advanced as a means for penumbral identification than MRI-based penumbral identification. However, as the thresholds of CBV and CBF needed to identify the ischemic core and hypoperfused region at risk of infarction become better characterized and the extent of tissue imaged is expanded, perfusion CT will evolve to a more widely used modality for penumbral identification in clinical trials and practice.

\section{AUTHOR CONTRIBUTIONS}

Dr. Fisher: drafting/revising the manuscript. Dr. Bastan: drafting/revising the manuscript, acquisition of data.

\section{DISCLOSURE}

Dr. Fisher serves on the scientific advisory boards for Photothera, CoAxia, Olea Medical, and Servier; received travel funding from Ferrer; served as Associate Editor of Stroke; serves as Editor-in-Chief of Stroke; served as consultant for Sygnis, Sanofi-Aventis, and Wyeth Pharmaceuticals; received honoraria from Mitsubishi Pharma USA and BioClinica; received research support from Pfizer and Sygnis; and holds stock options in Photothera and Brainsgate. Dr. Bastan reports no disclosures. Go to Neurology.org for full disclosures.

Received June 28, 2011. Accepted in final form August 31, 2011. 


\section{REFERENCES}

1. Astrup J, Siesjo BK, Symon L. Thresholds in cerebral ischemia: the ischemic penumbra. Stroke 1981;12:723-725.

2. Hossmann K-A. Viability thresholds and the penumbra of focal ischemia. Ann Neurol 1994;36:557-565.

3. Ginsberg MG, Pulsinelli WA. The ischemic penumbra; injury thresholds and the therapeutic time window for acute stroke. Ann Neurol 1994;36:553-554.

4. Hakim AM. The cerebral ischemic penumbra. Can J Neurol Sci 1997;14:557-559.

5. Fisher M. The ischemic penumbra: a new opportunity for neuroprotection. Cerebrovasc Dis 2006;21(suppl 2): 64-70.

6. Sharp FA, Lu A, Tang Y, Milhorn DE. Multiple molecular penumbras after focal cerebral ischemia. J Cereb Blood Flow Metab 2000;20:1011-1032.

7. Lo E. A new penumbra: transitioning from injury into repair after stroke. Nat Med 2008;14:497-500.

8. Datta A, Park JE, Li X, et al. Proteomic data mining showed upregulation. J Proteome Res 2010;9:472-484.

9. Lo E. Experimental models, neurovascular mechanisms and translational ischemic stroke research. Br J Pharmacol 2008;153:S396-S405.

10. Baron JC, Rougemont D, Soussaline F, et al. Local interrelationships of cerebral oxygen consumption and glucose utilization in normal subjects and ischemic stroke patients: a positron tomography study. J Cereb Blood Flow Metab 1984;4:140-149.

11. Baron J. Mapping the ischaemic penumbra with PET: implications for acute stroke treatment. Cerebrovasc Dis 1999;9:193-201.

12. Touzani O, Young AR, Derlon JM, et al. Sequential studies of severely hypometabolic tissue volumes after permanent middle cerebral artery occlusion: a positron emission tomography study in anesthetized baboons. Stroke 1995; 26:2112-2119.

13. Heiss WD, Graf R, Wienhard K, et al. Dynamic penumbra demonstrated by sequential multitracer PET after middle cerebral artery occlusion in cats. J Cereb Blood Flow Metab 1994;14:892-902.

14. Heiss WD. Ischemic penumbra: evidence from functional imaging in man. J Cereb Blood Flow Metab 2000;20: $1276-1293$

15. Heiss WD, Kracht LW, Thiel A, Grond M, Pawlik G. Penumbral probability thresholds of flumazenil binding and blood flow predicting outcome in patients with cerebral ischaemia. Brain 2001;124:20-29.

16. Read S, Hirano T, Abbott D, et al. The fate of hypoxic tissue on $18 \mathrm{~F}$-fluoromisonidazole positron emission tomography after ischemic stroke. Ann Neurol 2000;48: $228-235$.

17. Albers GW. Expanding the window for thrombolytic therapy in acute stroke: the potential role of acute MRI for patient selection. Stroke 1999;30:2230-2237.

18. Schlaug G, Benfield A, Baird AE, et al. The ischemic penumbra operationally defined by diffusion-perfusion MRI. Neurology 1999;53:1528-1537.

19. Calamante F, Thomas DL, Pell GS, Wiersma J, Turner R. Measuring cerebral blood flow using magnetic resonance imaging techniques. J Cereb Blood Flow Metab 1999;19: $701-735$.

20. Li F, Haw S, Tatlisumak T, et al. Reversal of acute apparent diffusion coefficient abnormalities and delayed neuro- nal death following transient focal cerebral ischemia in rats. Ann Neurol 1999;46:333-342.

21. Kidwell C, Saver J, Mattiello J. Thrombolytic reversal of acute human ischemic injury shown by diffusion/perfusion magnetic resonance imaging. Ann Neurol 2000;47:462469

22. Kane I, Carpenter T, Chappell F, et al. Comparison of 10 different magnetic resonance perfusion imaging processing methods in acute ischemic stroke. Stroke 2007;38:31583164

23. Henninger N, Sicard KM, Schmidt K, Bardutzky J, Fisher M. Comparison of ischemic lesion evolution in embolic vs. mechanical middle cerebral artery occlusion in SpragueDawley rats using diffusion and perfusion imaging. Stroke 2006;37:1283-1287.

24. Bratane B, Bouley J, Schneider A, et al. Granulocyte colony stimulating factor delays mismatch evolution and reduces infarct volume in permanent suture and embolic rat focal ischemia models. Stroke 2009;40:3102-3106.

25. Henninger N, Bratane BT, Bastan B, Bouley J, Fisher M. Normobaric hyperoxia and late tPA in a rat embolic stroke model. J Cereb Blood Flow Metab 2009;29:119-129.

26. Wintermark M, Bogousslavsky J. Imaging of acute ischemic brain injury: the return of computed tomography. Curr Opin Neurology 2003;16:59-63.

27. Wintermark M, Flanders AE, Velthuis B, et al. Perfusion-CT assessment of infarct core and penumbra. Stroke 2006;37: 979-985.

28. Murphy BD, Fox AJ, Sahlas DJ, et al. Identification of penumbra and infarct is acute ischemic stroke using computed tomography perfusion-derived blood flow and blood volume measurements. Stroke 2006;37:1771-1777.

29. Wintermark M, Meuli R, Browaeys P, et al. Comparison of CT perfusion and angiography and MRI in selecting stroke patients for treatment. Neurology 2007;68:694-697.

30. Ribo M, Molina CA, Rovira A, et al. Safety and efficacy of intravenous tissue plasminogen activator in the 3- to 6hour window using multimodal transcranial Doppler/ MRI selection protocol. Stroke 2005;36:602-606.

31. Schellinger PD, Gotz T, Fiehler J, et al. MRI and CT-based thrombolytic therapy in acute stroke within and beyond established time windows. Stroke 2007;38:2640-2645.

32. Albers GW, Thijs VN, Wechsler L, et al. Magnetic resonance imaging profiles predict clinical response to early reperfusion: the Diffusion and Perfusion Imaging Evaluation for Understanding Stroke Evolution (DEFUSE) study. Ann Neurol 2006;60:508-517.

33. Davis SM, Donnan GA, Parsons MW, et al. Effects of alteplase beyond $3 \mathrm{~h}$ after stroke onset in the Echoplanar Imaging Thrombolytic Evaluation Trial (EPITHET): a placebo-controlled randomized trial. Lancet Neurol 2008; 7:299-399.

34. Lansberg MG, Lee J, Christensen S, et al. RAPID automated patient selection for reperfusion therapy: a pooled analysis of the Echoplanar Imaging Thrombolytic Evaluation Trial (EPITHET) and the Diffusion and Perfusion Imaging Evaluation For Understanding Stroke Evolution (DEFUSE) study. Stroke 2011;42:1608-1614.

35. Hacke W, Albers GW, Al-Rawi Y, et al. The desmoteplase in acute ischemic stroke trial (DIAS): a phase II MRIbased 9-hour window acute stroke thrombolysis trial with intravenous desmoteplase. Stroke 2005;36:66-73.

36. Furlan AJ, Eyding D, Albers GW, et al. Escalation of Desmoteplase for Acute Ischemic Stroke (DEDAS): evidence 
of safety and efficacy 3 to 9 hours after stroke onset. Stroke 2006;37:1227-1231.

37. Hacke W, Furlan AJ, Al-Rawi Y, et al. Intravenous desmoteplase in patients with acute ischaemic stroke selected by MRI perfusion-diffusion weighted imaging or perfusion CT. Lancet Neurol 2009;8:141-150.

38. Warach S, for the DIAS 2 Study Group. Clinical benefit of desmoteplase treatment in patients with moderate to severe stroke-further results of the DIAS-2 study. Presented at the International Stroke Conference; 2008; New Orleans, LA.

39. Phan TG, Donnan GA, Davis SM, Byrnes G. Proof of principle phase II MRI studies in stroke: sample size estimates from dichotomous and continuous data. Stroke 2006;37:2521-2525.

40. Fisher M. New approaches to neuroprotective drug development. Stroke 2011;42:524-552. 


\title{
Neurology
}

\author{
Identifying and utilizing the ischemic penumbra \\ Marc Fisher and Birgul Bastan \\ Neurology 2012;79;S79-S85 \\ DOI 10.1212/WNL.0b013e3182695814
}

This information is current as of September 24, 2012

\section{Updated Information \& Services}

References

Citations

Subspecialty Collections

Permissions \& Licensing

Reprints including high resolution figures, can be found at: http://n.neurology.org/content/79/13_Supplement_1/S79.full

This article cites 39 articles, 16 of which you can access for free at: http://n.neurology.org/content/79/13_Supplement_1/S79.full\#ref-list-1

This article has been cited by 3 HighWire-hosted articles: http://n.neurology.org/content/79/13_Supplement_1/S79.full\#\#otherarti cles

This article, along with others on similar topics, appears in the following collection(s):

\section{CT}

http://n.neurology.org/cgi/collection/ct

Infarction

http://n.neurology.org/cgi/collection/infarction

MRI

http://n.neurology.org/cgi/collection/mri

Information about reproducing this article in parts (figures,tables) or in its entirety can be found online at:

http://www.neurology.org/about/about_the_journal\#permissions

Information about ordering reprints can be found online:

http://n.neurology.org/subscribers/advertise

Neurology ${ }^{\circledR}$ is the official journal of the American Academy of Neurology. Published continuously since 1951, it is now a weekly with 48 issues per year. Copyright Copyright (? 2012 by AAN Enterprises, Inc.. All rights reserved. Print ISSN: 0028-3878. Online ISSN: 1526-632X.

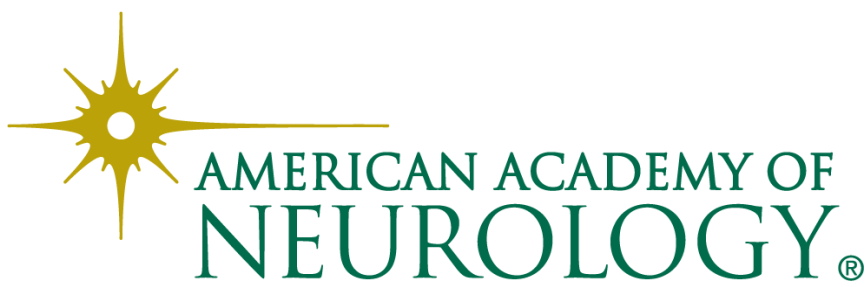

